\title{
Large deviation methods for stochastic
} reachability

Bujorianu, M.L. and Wang, Hong 2009

Manchester Institute for Mathematical Sciences

School of Mathematics

The University of Manchester

\footnotetext{
Reports available from: http://eprints.maths.manchester.ac.uk/

And by contacting: The MIMS Secretary

School of Mathematics

The University of Manchester

Manchester, M13 9PL, UK
} 


\title{
Large Deviation Methods for Stochastic Reachability
}

\author{
Manuela L. Bujorianu, Hong Wang
}

\begin{abstract}
In this paper, we propose to find upper/lower bounds for different measures that characterize the reachability problem defined in the context of stochastic hybrid systems, using the theory of large deviations. For stochastic hybrid processes, criteria for large deviation results are given using properties of their infinitesimal generators.. This represents just the first step towards applying large deviation methods for stochastic hybrid systems for treating new topics like robust control, metastability, performance analysis.
\end{abstract}

Keywords: stochastic hybrid systems, reachability, large deviations, Markov processes.

\section{INTRODUCTION}

Stochastic hybrid systems (SHS) are a class of non-linear stochastic continuous time/space hybrid dynamical systems. For these systems different models have been developed by many researchers in the field of hybrid systems. These models can be used to analyse and design complex embedded systems that operate in the presence of variability and uncertainty, and incorporate complex (hybrid/stochastic) dynamics, randomness, multiple modes of operations. Under some natural assumptions on their parameters, their behaviour can be described by stochastic processes having good properties. The verification problem for such systems consists in reachability analysis. The aim of reachability analysis is to determine the probability that the system will reach a set of desirable/unsafe states, and the difficulty of this problem comes from the interaction between discrete/continuous dynamics and the active boundaries.

In the literature, for deterministic hybrid systems there exist different methods to deal with the reachability problem. The most used methods are based on optimal control (Hamilton Jacobi equations) such that the computational issues are solved using dynamic programming. As well, reachability problem for hybrid systems can be thought of as an exit problem from a given domain. This also involves solving a standard Hamilton-Jacobi-Bellman equation over this set and possibly pieces of its boundary with rather complicated boundary conditions (see the discussions from [17] and the references therein).

In the SHS framework, it has been proved that aiming to tackle stochastic reachability as an optimal control problem could be a very challenging and difficult task. The main explanation for this difficulty can be found in the structure of the stochastic processes that describe the behaviour of SHS.

Manuela Bujorianu is with CICADA, School of Mathematics, University of Manchester, UK, E - mail : Manuela.Bujorianu@manchester.ac.uk

Hong Wang is with Control Systems Centre, School of Electrical and Electronics Engineering, University of Manchester, UK, E - mail :hong.wang@manchester.ac.uk
These processes are Markovian processes with piecewise continuous paths. Their discontinuities are describe by some spontaneous jumps (in a Poisson style) and forced jumps dictated by some guards. In mathematics these forced jumps are called predictable jumps. Their presence leads to some discontinuities of the transition probabilities of the Markov processes considered in this context. The main problem comes from the fact that the dynamic programming theory for the Markov processes (that describe the behaviour of SHS) with predictable jumps is not fully understood and developed. In most cases, dynamic programming methods are applied locally to these processes where they behave nicely like some diffusion processes or, more general, FellerMarkov processes [16]

For SHS, the stochastic reachability problem means to compute the probability of the set of those traces that start with a given probability distribution and hit in a finite/infinite horizon time a target set. In many papers, the standard methodology to approach this problem is to approximate the stochastic process that corresponds to the given hybrid system by simpler processes (like Markov chains) and then to derive convergence results for the reach set probabilities. Also, from a computer science perspective, Markov chain approximations are desirable for probabilistic model checking. Due to the complexity of such models, the Markov chain approximations suffer from state space explosion (see [16] and discussions therein). Then, at this point we are wondering what kind of approximations suit better to stochastic hybrid processes. Stochastic hybrid processes are jump type Markov processes. From the control theory and stochastic analysis perspectives, it seems that such processes are better studied using diffusion approximations [13]. Then the scope of approximations in this case is to "smooth" the process. For diffusion processes, very rich and powerful stochastic analysis tools are available. A natural research idea that comes from this debate is to develop "smooth" approximations for stochastic hybrid processes that can conduct to useful numerical methods for the computation of the reach set probabilities. But what is the meaning of "smooth" in this context? Intuitively, considering the discussions from the previous paragraph regarding the behaviour of SHS, "smooth" means that the forced transitions are removed. It is important to point out here that the first step in this direction has been done in [1]. It is not the aim of this work to extend [1], or to provide alternative methods for these smooth approximations. The main problem is to find the appropriate characterizations of the stochastic reachability, which, in an approximation scheme chosen "carefully" could be used straightforward to obtain convergence results. 
In this paper, we characterize stochastic reachability using expectations of the hitting times for the target sets. The main idea is that the reachability problem can be treated as an exit problem. In the first case, it can be proved that the reach set probability is exactly the expectation of the probabilistic event that the first hitting time of the target set (or the exit time from the complement of this set) is less than the horizon time. The quantities involved can be characterised as solutions for some appropriate Hamilton Jacobi equations [10].

In the mathematical literature, the problem of computing the escape rate and of the probability distribution of the escape points on the boundary of a given domain is referred to as Kolmogorov's exit problem. The first result on this problem seems to be the "Large Deviation Theory (LDT)" result of Wentzell and Freidlin [11]. Large deviation is a part of probability theory that provides asymptotic estimates for probabilities of rare events. Roughly speaking, LDT concerns itself with the exponential decay of the probability measures of certain kinds of extreme or tail events, as the number of observations grows arbitrarily large. A unifying framework for the LDT has been developed by S.R.S. Varadhan [20]. A rather general singular perturbation method for the calculation of both the escape rate and the escape distribution was introduced in [19]. The method of [19] was based on the construction of uniform asymptotic expansions of the solutions of the mean first passage time equation and of the stationary Fokker-Planck equation. For jump Markov processes an asymptotic method has been developed in [15]. There exists a very rich literature [9] regarding the asymptotic estimations for exit time probabilities associated with different classes of Markov processes. This provides us techniques, ideas and methodologies that can help in dealing with the exit problems for SHS. Moreover, many of these classes of Markov processes can be considered particular stochastic hybrid processes (Markov processes with Levy generators, dynamical systems driven by some Markov jump processes, jump-diffusions). The main goal in these paper is to consider asymptotic expansions of certain expectations of the Markov processes under study and to find upper/lower bounds or exact analytical solutions of quantities of interest (mean exit times, quasistationary distributions, Feynman-Kac functionals). The techniques are based on results from LDT. Then, in the framework of SHS, a natural idea is to consider a family of scaled processes with respect to some $\epsilon>0$ (asymptotic approximations) for a stochastic hybrid process satisfying the hypotheses of LDT and to derive results for reach set probabilities.

\section{PRELIMinaries}

\section{A. Markov Processes}

Let us consider $M=\left(x_{t}, P_{x}\right)$ a Markov process with the state space $X$. A Markov process retains no memory of where it has been in the past. Standard definitions can be find in any textbook [7]. We adjoin an extra point $\Delta$ (the cemetery) to $X$ as an isolated point, $X_{\Delta}=X \cup\{\Delta\}$. The existence of $\Delta$ is assumed in order to have a probabilistic interpretation of $P_{x}\left(x_{t} \in X\right)<1$, i.e. at some 'termination time' $\zeta(\omega)$ when the process $M$ escapes to and is trapped at $\Delta$.

$X$ is equipped with Borel $\sigma$-algebra $\mathcal{B}(X)$ or shortly $\mathcal{B}$. Consider the set $\mathbf{B}(X)$ of bounded real measurable functions defined on $X$, which is a Banach space with the sup-norm $\|\varphi\|=\sup _{x \in X}|\varphi(x)|, \varphi \in \mathbf{B}(X)$. Suppose we have given a $\sigma$-finite measure $\mu$ on $(X, \mathcal{B})$.

The semigroup of operators $\mathcal{P}=\left(P_{t}\right)$ is given by

$$
P_{t} f(x)=E_{x} f\left(x_{t}\right)=\int f(y) p_{t}(x, d y), t \geq 0
$$

where $E_{x}$ is the expectation with respect to $P_{x}$ and $p_{t}(x, A)$, $x \in X, A \in \mathcal{B}$ represent the transition probabilities, i.e. $p_{t}(x, A)=P_{x}\left(x_{t} \in A\right)$. The semigroup property of $\left(P_{t}\right)$ can be derived from the Chapman-Kolmogorov equations satisfied by the transition probabilities. The infinitesimal generator of $M$, denoted by $\mathcal{L}$, is the derivative of $P_{t}$ at $t=0$. Let $D(\mathcal{L}) \subset \mathcal{B}^{b}(X)$ be the set of functions $f$ for which the following limit exists (denoted by $\mathcal{L} f$ )

$$
\lim _{t \searrow 0} \frac{1}{t}\left(P_{t} f-f\right)
$$

In most cases, the operator semigroup can be itself characterized by its infinitesimal generator. When $D(\mathcal{L})$ is large enough, the infinitesimal generator captures the law of the whole dynamics of a Markov process and provides a tool to study the Markov process.

The operator resolvent $\mathcal{V}=\left(V_{\alpha}\right)_{\alpha \geq 0}$ associated with $\mathcal{P}$ is the Laplace transform of the semigroup.

A quadratic form $\mathcal{E}$ can be associated to the generator of a Markov process in a natural way. Let $L^{2}(X, \mu)$ be the space of square integrable $\mu$-measurable extended real valued functions on $X$, w.r.t. the natural inner product $\langle f, g\rangle_{\mu}=$ $\int f(x) g(x) d \mu(x)$.

Quadratic form $\mathcal{E}$ :

$$
\mathcal{E}(f, g)=-<\mathcal{L} f, g>_{\mu}, f \in D(\mathcal{L}), g \in L^{2}(X, \mu)
$$

(3) defines a closed form. This leads to another way of parameterizing Markov processes. Instead of writing down a generator one starts with a quadratic form. As in the case of a generator it is typically not easy to fully characterize the domain of the quadratic form. For this reason one starts by defining a quadratic form on a smaller space and showing that it can be extended to a closed form in subset of $L^{2}(\mu)$. When the Markov process can be initialized to be stationary, the measure $\mu$ is typically this stationary distribution (see [7], p.111). More generally, $\mu$ does not have to be a finite measure.

A coercive closed form is a quadratic form $(\mathcal{E}, D(\mathcal{E}))$ with $D(\mathcal{E})$ dense in $L^{2}(X, \mu)$, which satisfies the:

(i) closeness axiom, i.e. its symmetric part is positive definite and closed in $L^{2}(X, \mu)$, (ii) continuity axiom.

$\mathcal{E}$ is called Dirichlet form if, in addition, it satisfies the third axiom:

(iii) contraction condition, i.e. $\forall u \in D(\mathcal{E}), u^{*}=u^{+} \wedge 1 \in$ $D(\mathcal{E})$ and $\mathcal{E}\left(u \pm u^{*}, u \mp u^{*}\right) \geq 0$. 
For a the general theory of closed forms associated with Markov processes see [18]. Let $(\mathcal{L}, D(\mathcal{L}))$ be the generator of a coercive form $(\mathcal{E}, D(\mathcal{E}))$ on $L^{2}(X, \mu)$, i.e. the unique closed linear operator on $L^{2}(X, \mu)$ such that $1-\mathcal{L}$ is onto, $D(\mathcal{L}) \subset D(\mathcal{E})$ and $\mathcal{E}(u, v)=<-\mathcal{L} u, v>$ for all $u \in D(\mathcal{L})$ and $v \in D(\mathcal{E})$. Let $\left(T_{t}\right)_{t>0}$ be the strongly continuous contraction semigroup on $L^{2}(X, \mu)$ generated by $\mathcal{L}$ and $\left(G_{\alpha}\right)_{\alpha>0}$ the corresponding strongly continuous contraction semigroup (which exist according to the HilleYosida theorem). A right process $M$ with the state space $X$ is associated with a Dirichlet form $(\mathcal{E}, D(\mathcal{E}))$ on $L^{2}(X, \mu)$ if the semigroup $\left(P_{t}\right)$ of the process $M$ is a $\mu$-version ${ }^{1}$ of the form semigroup $\left(T_{t}\right)$.

\section{STOChASTIC HybRID SYSTEMS}

We adopt the General Stochastic Hybrid System model presented in [4]. This subsection describes the model and establishes the notation.

Let $Q$ be a set of discrete states. For each $q \in Q$, we consider the Euclidean space $\mathbb{R}^{d(q)}$ with dimension $d(q)$ and we define an invariant as an open subset $X^{q}$ of $\mathbb{R}^{d(q)}$. The hybrid state space is the set $X(Q, d, \mathcal{X})=\bigcup_{i \in Q}\{i\} \times X^{i}$ and $x=\left(i, z^{i}\right) \in X(Q, d, \mathcal{X})$ is the hybrid state. The closure of the hybrid state space will be $\bar{X}=X \cup \partial X$, where $\partial X=\bigcup_{i \in Q}\{i\} \times \partial X^{i}$. It is known that $X$ can be endowed with a metric $\rho$ whose restriction to any component $X^{i}$ is equivalent to the usual component metric [7]. Then $(X, \mathcal{B}(X))$ is a Borel space (homeomorphic to a Borel subset of a complete separable metric space), where $\mathcal{B}(X)$ is the Borel $\sigma$-algebra of $X$. Let $\mathbf{B}(X)$ be the Banach space of bounded positive measurable functions on $X$ with the norm given by the supremum.

A (General) Stochastic Hybrid System (SHS) is a collection

$$
H=\left((Q, d, \mathcal{X}),(b, \sigma), \mu_{0},(\lambda, R)\right)
$$

where

- $(Q, d, \mathcal{X})$ describes the hybrid state space: $Q$ is a countable/finite set of discrete states (modes); $d: Q \rightarrow \mathbb{N}$ is a map giving the dimensions of the continuous state spaces; $\mathcal{X}: Q \rightarrow \mathbb{R}^{d(.)}$ maps each $q \in Q$ into an open subset $X^{q}$ of $\mathbb{R}^{d(q)}$

- $(b, \sigma)$ provides the coefficients of the diffusion part (continuous dynamics in modes): $b: X(Q, d, \mathcal{X}) \rightarrow \mathbb{R}^{d(.)}$ is a vector field; $\sigma: X(Q, d, \mathcal{X}) \rightarrow \mathbb{R}^{d(\cdot) \times m}$ is a $X^{(\cdot)}$-valued matrix, $m \in \mathbb{N}$,

- $\mu_{0}$ is the initial probability measure defined on $(X, \mathcal{B}(X))$; - $(\lambda, R)$ gives the jumping mechanism: $\lambda: \bar{X}(Q, d, \mathcal{X}) \rightarrow$ $\mathbb{R}^{+}$is a transition rate function; $R: \bar{X} \times \mathcal{B}(\bar{X}) \rightarrow[0,1]$ is a stochastic kernel that provides the post-jump location.

The realization of an SHS is built as a Markov string [4] obtained by the concatenation of the paths of some diffusion processes $\left(z_{t}^{i}\right), i \in Q$ together with a jumping mechanism given by a family of stopping times $\left(S^{i}\right)$.

\footnotetext{
${ }^{1}$ I.e., for all $f \in L^{2}(X, \mu)$ the function $P_{t} f$ is a $\mu$-version (differs on a set of $\mu$-measure zero) of $T_{t} f$ for all $t>0$.
}

A stochastic process $x_{t}=(q(t), z(t))$ is called an $S H S$ realization if there exists a sequence of stopping times $T_{0}=$ $0<T_{1}<T_{2} \leq \ldots$ such that for each $k \in \mathbb{N}$,

- $x_{0}=\left(q_{0}, z_{0}^{q_{0}}\right)$ is a $Q \times X$-valued random variable chosen according to the probability distribution $\mu_{0}$;

- For $t \in\left[T_{k}, T_{k+1}\right), q_{t}=q_{T_{k}}$ is constant and $z(t)$ is a solution of the stochastic differential equation (SDE):

$$
d z(t)=b\left(q_{T_{k}}, z(t)\right) d t+\sigma\left(q_{T_{k}}, z(t)\right) d W_{t}
$$

where $W_{t}$ is a the $m$-dimensional standard Wiener process; - $T_{k+1}=T_{k}+S^{i_{k}}$ where $S^{i_{k}}$ is chosen according to the survivor function $F$.

- The post jump location $x\left(T_{k+1}\right)$ is sampled according to the probability distribution $R\left(\left(q_{T_{k}}, z\left(T_{k+1}^{-}\right)\right), \cdot\right)$.

The realization of any SHS, $H$, under standard assumptions (about the diffusion coefficients, non-Zeno executions, transition measure, etc, see [4] for a detailed presentation) is a strong Markov process. Let $M=\left(\Omega, \mathcal{F}, \mathcal{F}_{t}, x_{t}, P_{x}\right)$ be the strong Markov process associated to $H$. The sample paths of $M$ are right continuous with left limit, i.e. cadlags.

Given a function $f \in \mathcal{C}^{1}\left(\mathbb{R}^{n}, \mathbb{R}\right)$ and a vector field $b: \mathbb{R}^{n} \rightarrow \mathbb{R}^{n}$, we use $\mathcal{L}_{b} f$ to denote the Lie derivative of $f$ along $b$ given by $\mathcal{L}_{b} f(x)=\sum_{i=1}^{n} \frac{\partial f}{\partial x_{i}}(x) f_{i}(x)$. Given a function $f \in \mathcal{C}^{2}\left(\mathbb{R}^{n}, \mathbb{R}\right)$, we use $\mathbb{H}^{f}$ to denote the Hamiltonian operator applied to $f$, i.e. $\mathbb{H}^{f}(x)=\left(h_{i j}(x)\right)_{i, j=1 \ldots n} \in$ $\mathbb{R}^{n \times n}$, where $h_{i j}(x)=\frac{\partial^{2} f}{\partial x_{i} \partial x_{j}}(x) \cdot A^{T}$ denotes the transpose matrix of a matrix $A=\left(a_{i j}\right)_{i, j=1 \ldots n} \in \mathbb{R}^{n \times m}$ and $\operatorname{Tr}(A)$ denotes its trace. The infinitesimal generator of an SHS is an integro-differential operator. The extended generator of an SHS has the following expression:

$$
\mathcal{L} f(x)=\mathcal{L}_{\text {cont }} f(x)+\lambda(x) \int_{\bar{X}}(f(y)-f(x)) R(x, d y)
$$

where $\mathcal{L}_{\text {cont }} f(x)$ has the standard form of the diffusion infinitesimal operator:

$$
L_{\text {cont }} f(x)=\mathcal{L}_{b} f(x)+\frac{1}{2} \operatorname{Tr}\left(\sigma(x) \sigma(x)^{T} \mathbb{H}^{f}(x)\right) .
$$

What makes this generator different from the generator of a Feller Markov process (like a diffusion process) is its domain that contains at least the set of second order differentiable functions that satisfy the boundary condition, as follows: $f(x)=\int_{\mathbb{X}} f(y) R(x, d y), x \in \partial X$. In the presence of forced jumps, the generator of an SHS is an operator that is difficult to deal with, since its domain does not even contain the set of all compactly supported $C^{\infty}$ functions.

\section{Stochastic Reachability}

\section{A. Definitions}

Let us consider $M=\left(\Omega, \mathcal{F}, \mathcal{F}_{t}, x_{t}, P_{x}\right)$ being a (strong right) Markov process, the realization of an SHS $H$. We address the following stochastic reachability problem. Given a target set, the objective of the reachability problem is to compute the probability that the system trajectories from an arbitrary initial state will reach the target set. Formally, given a set $A \in \mathcal{B}(X)$ and a time horizon $T>0$, let us define:

$\operatorname{Reach}_{T}(A)=\left\{\omega \in \Omega \mid \exists t \in[0, T]: x_{t}(\omega) \in A\right\}$ 
$\operatorname{Reach}_{\infty}(A)=\left\{\omega \in \Omega \mid \exists t \geq 0: x_{t}(\omega) \in A\right\}$.

These two sets are the sets of trajectories of $M$, which reach the set $A$ (the flow that enters $A$ ) in the interval of time $[0, T]$ or $[0, \infty)$. The reachability problem consists of determining the probabilities of such sets. The probabilities of reach events are

$$
P\left(T_{A}<T\right) \text { or } P\left(T_{A}<\zeta\right)
$$

where $\zeta$ is the life time of $M$ and $T_{A}$ is the first hitting time of $A$

$$
T_{A}=\inf \left\{t>0 \mid x_{t} \in A\right\}
$$

and $P$ is a probability on the measurable space $(\Omega, \mathcal{F})$ of the elementary events associated to $M . P$ can be chosen to be $P_{x}$ (if we want to consider the trajectories that start in $x$ ) or $P_{\mu_{0}}$ ((if we want to consider the trajectories with an initial condition chosen according to an initial probability distribution $\mu_{0}$ ).

Denote by $P_{A}$ the hitting operator associated to the underlying Markov process $\left(x_{t}\right)$, i.e.

$$
P_{A} v=E_{x}\left\{v \circ x_{T_{A}} \mid T_{A}<\zeta\right\}
$$

and $T_{A}$ is given by (8).

Proposition 1: [5] For any $x \in X$ and Borel set $A \in$ $\mathcal{B}(X)$, we have $P_{x}\left[\operatorname{Reach}_{\infty}(A)\right]=P_{A} 1(x)=P_{x}\left[T_{A}<\zeta\right]$.

\section{B. Exit Problem}

Note that the first hitting time of $A$ is equal with the first exit time from the complementary set of $A, E=A^{c}=X \backslash A$. Then, the stochastic reachability problem can be formulated as an exit problem for the right Markov processes that appear as realizations of SHS. These processes may be viewed as piecewise continuous jump diffusions, where the jumps are allowed to be spontaneous, or forced (predictable). For continuous pure diffusions processes, it is sufficient to consider the time when the process hits the boundary of $E$ or $A$. However, when the stochastic processes also includes jumps, then it is possible that the process overshoots the boundary and ends up in the exterior of the domain $E$ (i.e. in the interior of $A$ ).

An important quantity that can be computed without explicitly constructing the transition probability density function is the mean first passage time of the process from a specified domain. The mean first passage time is a measure of the stochastic time scale for the process to be in a specified domain. The mean first passage time is a solution of a boundary value problem involving the backward Kolmogorov operator (the adjoint of the operator in the forward equation). If the process is a continuous diffusion with the infinitesimal generator $L$ and the target set $A$ is a closed set, it is known that if the PDE (Dirichlet problem)

$$
\begin{cases}\frac{\partial u}{\partial t}=L u & \text { on } E \times(0, T] \\ u=0 & \text { on } E \times\{0\} \\ u=\mathbf{1}_{\partial E} & \text { on } \partial E \times(0, T]\end{cases}
$$

has a bounded solution, then

$$
u(x, t)=P_{x}\left\{T_{A} \leq t, x_{T_{A}} \in \partial E\right\}, 0 \leq t \leq T .
$$

Let us consider an SHS $H$ = $\left((Q, d, \mathcal{X}),(b, \sigma), \mu_{0},(\lambda, R)\right)$ defined as in the Section III, with a finite number of modes $\left(\operatorname{card} Q<\aleph_{0}\right)$. We can suppose that $H$ has only forced jumps, no spontaneous jumps. This can be achieved introducing an extra variable that "simulates" the spontaneous jumps [7].

Suppose that the target set $A$ is closed and let $E$ be its complement. Denote $E^{q}=X^{q} \cap E, q \in Q$. Then $\left\{\partial E^{q} \mid q \in\right.$ $Q\}$ represents a partition of the boundary $\partial E$. Suppose that supp $\mu_{0} \subset E$ ( $\mu_{0}$ is the initial probability measure). The first quantities we need to compute are:

$u^{q}(t)=P_{x}\left(T_{A}<t, x_{T_{A}} \in \partial E^{q}\right\}, q \in Q, 0 \leq t \leq T$.

Then, for each $q \in Q, u^{q}$ is solutions of the Dirichlet problem associated to the diffusion process associated to the mode $q$ (see the previous paragraph).

\section{Occupation time distribution}

For a sample path $\omega$, we define the occupation time distribution up to the time $t>0$ as follows:

$$
L_{t}(\omega, B)=\frac{1}{t} \int_{0}^{t} 1_{B}\left(x_{s}(\omega)\right) d s, B \in \mathcal{B}(X) \text {. }
$$

If the process is conservative, then $L_{t}(\omega, \cdot)$ is a probability measure on $(X, \mathcal{B})$.

Remark 1: One way to deal with stochastic reachability for a target set $A$, is to find how the expectation of the empirical measure defined by (11) corresponding to the complementary set $E$ of $A$ differs from the distribution of $E$.

\section{Large Deviation Methods for SHS}

The area of large deviations is a set of asymptotic results on rare event probabilities and a set of methods to derive such results. In this section, we formally define the concept of "smooth" approximations for SHS. Based on the theory of large deviations, we give some convergence results for reach set probabilities.

\section{A. Definitions}

Large deviations theory is concerned with asymptotic estimates of rare event probabilities associated with stochastic processes. Let us consider $A^{\epsilon}$ an event associated with some stochastic process $x_{t}^{\epsilon}$ with exponentially small probability,.i.e.

$$
-\lim _{\epsilon \rightarrow 0} \epsilon \log P\left(A^{\epsilon}\right)=V^{0}
$$

When $P\left(A^{\epsilon}\right)$ satisfies (12), then $A^{\epsilon}$ is said to have a large deviations property and $V^{0}$ is the large deviation rate. In a similar way, one could seek a large deviation property for the expectations $E \Phi^{\epsilon}\left(x^{\epsilon}\right)$ expressed by $-\lim _{\epsilon \rightarrow 0} \epsilon \log E \Phi^{\epsilon}\left(x^{\epsilon}\right)=V^{0}$.

Large deviation results are closely linked with modifications of the probability measure $P$, under which the event $A^{\epsilon}$ is rare. This event is no longer rare under a new probability $P^{\epsilon}$ that is a change of $P$. Usually, the Radon-Nikodym derivative $d P / d P^{\epsilon}$ has an exponential form. When $\epsilon$ is small, it is necessary to find the contribution to the exponent. If $x_{t}$ is 
a continuous time Markov process, then suitable change from $P$ to $P^{\epsilon}$ is typically the result of a change of its generator.

At this stage, it is convenient to introduce the Varadhan unifying framework for LDT. The idea is to characterise the limiting behaviour of a family $\left(P_{\epsilon}\right)$ of probability measures as $\epsilon \searrow 0$ in terms of a rate function. Let $(X, d)$ be a complete separable metric space, and $\mathcal{B}$ denotes its Borel $\sigma$-algebra.

A function $I: X \rightarrow[0, \infty]$ is called rate function if $I$ is not identic with $\infty$ and if the level set $\{x \in X \mid I(x) \leq c\}$ is compact in $X$ for each $c<\infty$. In particular, a rate function is lower semicontinuous (1.s.c.), i.e. $I^{-1}([0, c])$ is closed in $X$ for all $c<\infty$.

Let $\left\{P_{\epsilon}: \epsilon>0\right\}$ be a family of probability measures on $(X, \mathcal{B})$. The family $\left\{P_{\epsilon}\right\}$ is said to satisfy the large deviation principle $(L D P)$ with rate function $I$ if

(i) $I$ is a rate function,

(ii) for every closed set $F \subseteq X$,

$\lim \sup _{\epsilon \rightarrow 0} \epsilon \log P_{\epsilon}(F) \leq-\inf _{y \in F} I(y)$,

(iii) for every open set $G \subseteq X$,

$\liminf _{\epsilon \rightarrow 0} \epsilon \log P_{\epsilon}(G) \geq-\inf _{y \in G} I(y)$.

LDP constitutes an important mechanism that can be employed for proving approximation results for different quantities of interest (mean exit times, quasistationary distributions, Feynman-Kac functionals) associated to a stochastic process.

\section{B. LDT for SHS}

In order to prove that the realization of an SHS satisfies the LDP, one must establish an upper bound for closed sets, and a lower bound for open sets. The purpose of this subsection is to establish the upper large deviation bound for SHS realizations that belong to a general class of Markov processes taking values in the Skorohod space $D([0, T], X)$ (the space of cadlag functions in $X)$. We follow the methodology proposed in [8].

Let $H$ be an SHS defined as in Section III. The following assumptions will be in force:

Assumption 1: For each $x \in X$, the measure $R(x, \cdot)$ has compact support.

For $\epsilon>0$, we define an operator $L^{\epsilon}$ on twice continuously differentiable functions by the formula:

$$
L^{\epsilon} f(x)=\sum_{i=1}^{n} b_{i}(x) \frac{\partial f}{\partial x_{i}}+\frac{\epsilon}{2} \operatorname{Tr}\left(\sigma(x) \sigma(x)^{T} \mathbb{H}^{f}(x)\right)+
$$
$\frac{1}{\epsilon} \lambda(x) \int_{\bar{X}}(f(x+\epsilon y)-f(x)) R(x, d y)$

Assumption 2: For $T>0$, there exists a Markov process $\left\{x^{\epsilon}(t) \mid 0 \leq t \leq T\right\}$ corresponding to the infinitesimal $L^{\epsilon}$ (in the sense that the martingale problem is well posed for this operator) with right continuous with left limits paths,

For general Markov processes, the LDP has been proven to hold only partially (with some upper bounds).

Some notations are needed, as follows. For $x, \alpha \in \mathbb{R}^{n}$, define

$$
\begin{aligned}
H(x, \alpha):=<b(x), \alpha>+\frac{1}{2}<\sigma(x) \sigma(x)^{T} \alpha, \alpha> \\
+\lambda(x) \int_{\bar{X}} \exp (<\alpha, y>-1) R(x, d y)
\end{aligned}
$$

and the upper semicontinuous regularization of $H(\cdot, \alpha)$ as $h(x, \alpha)=\lim _{\delta \searrow 0} \sup _{|y-x| \leq \delta} H(y, \alpha)$. Consider the Legendre-Frenchel transform: $l(x, \beta)=\sup _{\alpha \in \mathbb{R}^{n}}[\alpha \cdot \beta-$ $h(x, \alpha)], \beta \in \mathbb{R}^{n}$. In terms, of this, we define the functional $I_{x}(\phi)=\int_{0}^{T} l(\phi(s), \dot{\phi}(s)) d s$, when $\phi$ is absolutely continuous and $\phi(0)=x$. In all other cases, we set $I_{x}(\phi)=+\infty$.

Theorem 2: For $T>0$ and $\epsilon>0$, we suppose that the Assumptions 1, 2 are in force. Let $K$ a compact subset of $X$. Then the following conclusions hold:

(i) Define $\Phi_{x}(L)=\left\{\phi \in D([0, T], X): I_{x}(\phi) \leq L\right\}$. Then for all $L<\infty$, the set $\cup_{x \in K} \Phi_{x}(L)$ is compact.

(ii) For each closed set $F$ in $D([0, T], X)$ we have $\limsup \epsilon \log P_{x}\left(x_{t}^{\epsilon} \in F\right\} \leq-\inf _{\phi \in F} I_{x}(\phi)$ uniformly in $x \in K$.

\section{LARge DeViation Methods for Stochastic REACHABILITY}

\section{A. LDT for reach set probabilities}

Let us consider $M=\left(x_{t}, P_{x}\right)$ be the strong Markov process associated to an SHS $H$ defined on the hybrid state space $X . X$ can be embedded in an Euclidean space $\mathbb{R}^{n}$. Let $T>0$ an horizon time and $A$ a target set such that $E=A^{c}$ is unbounded. Let $\epsilon>0$ denote a small parameter. For $M$, we consider a family of scaled processes $\left(x_{t}^{\epsilon}\right)$ generated by $\left.\left(x_{t}\right):\left(x_{t}^{\epsilon} \mid t \in[0, T]\right):=\left(\epsilon x_{t / \epsilon}\right) \mid t \in[0, T]\right)$. The process $\left(x_{t}\right)$ will be assumed to satisfy the following conditions:

(H1) Sample path large deviations. For every $T>0$, the family of rescaled processes $\left(x_{t}^{\epsilon} \mid t \in[0, T]\right)$ satisfy sample path large deviation principle in the Skorohod space $D([0, T], X)$ with a good rate function $I_{[0, T]}$. Define for any $x=(q, z), x^{\prime}=\left(q^{\prime}, z^{\prime}\right), I_{T}\left(x, x^{\prime}\right)$ as the infimum of the rate $I_{[0, T]}(\phi)$ over all $\phi \in D([0, T], X)$ with $\phi(0)=x$ and $\phi(T)=x^{\prime}$.

(H2) Asymptotically finite range. The function

$$
\widehat{\varphi}(\alpha):=\sup _{x \in E} \sup _{t \in[0,1]} E_{x}[\exp (\alpha(Z(t)-x))]
$$

is finite everywhere on $\mathbb{R}^{n}$.

Let $\mathcal{R}$ denote the set of all possible limits $\lim _{\epsilon \rightarrow 0} \epsilon x_{\epsilon}$ with $x_{\epsilon} \in E$. For given $x, x^{\prime} \in \mathcal{R}$ we let $I\left(x, x^{\prime}\right):=$ $\inf _{T>0} I_{T}\left(x, x^{\prime}\right)$.

$$
\widehat{I}\left(x, x^{\prime}\right):= \begin{cases}I\left(x, x^{\prime}\right) & \text { if } x \neq x^{\prime} \\ 0 & \text { if } x=x^{\prime}\end{cases}
$$

Theorem 3: Suppose that the conditions (H1) and (H2) are satisfied and let $I_{T}\left(x_{0}, x_{0}\right)>0$. Then the following assertions hold:

(i) for any $x \in \mathcal{R}$ and any open set $G \subset X$ : $\liminf _{\epsilon \rightarrow 0} \epsilon \ln u^{\epsilon}(x, T) \geq-\inf _{x^{\prime} \in G} I\left(x, x^{\prime}\right)$;

(ii) for any $x \in \mathcal{R}$ and any compact set $K \subset$ $X: \limsup _{\epsilon \rightarrow 0} \epsilon \ln u^{\epsilon}(x, T) \leq-\inf _{x^{\prime} \in K} I\left(x, x^{\prime}\right)$, where $u^{\epsilon}(x, t)$ is the reach set probability function given by (10) for the process $\left(x_{\epsilon}\right)$.

\section{B. Sanov theorem for the occupation time distribution}

Let us consider a different class of large deviations problems, which belong to the type introduced by Donsker and Varadhan. Let $x_{t}$ be a stochastic process, considered for $0 \leq t \leq T$ where $T$ is finite but large. The role of small parameters $\epsilon$ is now taken by $T^{-1}$. In this case, methods 
to construct good rates for the large deviation principle use mainly the infinitesimal generator of the process (see [12] and the references therein).

In this subsection, we use good rate functions for strong Markov processes written in terms of their Dirichlet forms associated (see [22] and the references therein). The expression of the Dirichlet form associated to a realization of an SHS can be easily derived using the expression of the generator 5. Moreover, this Dirichlet form can be expressed also using Beurling-Denny formula [18], which illustrates clearly the continuous part and the jumping part of the process.

The symmetrized Dirichlet form corresponding to $\mathcal{E}$ defined by (3) is given by $\mathcal{E}^{\sigma}(f, g):=1 / 2\left[<-\mathcal{L} f, g>_{\mu}\right.$ $\left.+<-\mathcal{L} g, f>_{\mu}\right], \forall f, g \in D_{2}(\mathcal{L})$, where $D_{2}(\mathcal{L}):=$ $D(\mathcal{L}) \cap L^{2}(X, \mu)$. Let us define $J_{E}(r):=\inf \left\{\mathcal{E}^{\sigma}(f, f) \mid f \in\right.$ $\left.D_{2}(\mathcal{L}), \int f^{2} d \mu=1 ; \int_{E} f^{2} d \mu=r\right\}$, for every $r \in \mathbb{R}$ (convention: $\inf \emptyset:=+\infty$ ). Clearly, $J_{E}$ is a convex function. Consider $I_{E}$ the lower semi-continuous (1.s.c.) regularization of $J_{E}$.

Theorem 4: For any initial measure $\nu$ such that $\nu \ll \mu$ and its Radon Nikodym derivative $\rho=\frac{d \nu}{d \mu} \in L^{2}(X, \mu)$, we have for all $t>0$, all $r>0$

$$
\begin{aligned}
P_{\nu}\left(L_{t}(\omega, E)\right. & >\mu(E)+r) \leq \\
& \leq\|\rho\|_{L^{2}} \exp \left(-t \cdot I_{E}(\mu(E)+r)\right), \\
P_{\nu}\left(L_{t}(\omega, E)\right. & <\mu(E)-r) \leq \\
& \leq\|\rho\|_{L^{2}} \exp \left(-t \cdot I_{E}(\mu(E)-r)\right)
\end{aligned}
$$

Remark 2: In the context of stochastic reachability, the above theorem states that the occupation time distribution of the complement of the target set lies in a certain vicinity of its mean, whose bounds are related through $J_{E}$ with the equilibrium potential of this set.

\section{Asymptotic expansions}

In the previous subsections, we have given the general framework for asymptotic approximations of stochastic hybrid processes and the convergence result for the reach set probabilities. Examples and particular cases of SHS have been already studied in the literature: for Markov Jump Processes see [15]; for one-dimensional Piecewise Deterministic Markov Processes see [14]; for Markov processes with Levy generators see [9]; for diffusions with jumps see [21]. Examples from these papers illustrate the effectiveness of this method.

\section{CONCLUSIONS}

In this paper, we have developed large deviation methods to approach the reachability problem for SHS. The starting point was to present the stochastic reachability problem as an exit problem for Markov processes with piecewise diffusion behavior and forced jumps. Moreover, the problem of reachability can be treated using not only hitting time probabilities, but also other measures like the distribution of the occupation time of a specific set, or the Green operator associated to the corresponding Markov process. A corner stone of this approach is to identify the right hypotheses such that the large deviation methods developed in the literature can be applied to SHS. The relevance of large deviation methods for stochastic reachability should not be judged in the context of simulation approaches for stochastic reachability. Rare event simulation methods (like importance sampling, importance splitting) SHS are treated by different other papers as [3]. We have provided large deviation methods for different measures (exit probabilities, occupation time distributions) associated to the stochastic reachability problem. These methods employ good function rates that can be computed in terms of the infinitesimal generator of a stochastic hybrid process and offer lower/upper bounds for the quantities that characterize the reachability problem.

\section{REFERENCES}

[1] Abate, A., Prandini, M., Lygeros, J., Sastry, S.: Approximation of General Stochastic Hybrid Systems by Switching Diffusions with Random Hybrid Jumps. HSCC (2008): 598-601

[2] Blom, H.A.P., Lygeros, J. (Eds.): "Stochastic Hybrid Systems: Theory and Safety Critical Applications". LNCIS 337 (2006).

[3] Blom, H.A.P.; Bakker, G.J.; Krystul, J.: Probabilistic reachability analysis for large scale stochastic hybrid systems. Proc. 46th Conference in Decision and Control (2007): 3182 - 3189.

[4] Bujorianu, M.L., Lygeros, J.: Towards Modelling of General Stochastic Hybrid Systems. In [2]: 3-30.

[5] Bujorianu, M.L., Lygeros, J.: New Insights on Stochastic Reachability. Proc. 46th Conference in Decision and Control (2007).

[6] Donsker, M. D. and Varadhan, S. R. S.: Asymptotic evaluation of certain Markov process expectations for large time. IV. Comm. Pure. Appl. Math. (36) (1983).

[7] Davis, M.H.A.: "Markov Models and Optimization" Chapman \& Hall, (1993).

[8] Dupuis, P., Ellis, R., Weiss, A.: Large Deviations for Markov Processes with Discontinuous Statistics I: General Upper Bounds. Ann. Prob.(19) (1991): 1280-1297.

[9] Fleming, W.H., Soner, H.M.: Asymptotic Expansions for Markov Processes with Levy Generators. Appli. Math. Optim. (19) (1989): 203-223.

[10] Fleming, W.H.: Exit probabilities and optimal stochastic control. Applied. Math. Optim. (4) (1978): 329-346.

[11] Freidlin, M.I., Wentzell, A.D.: "Random Perturbations of Dynamical Systems ". Springer-Verlag (1969).

[12] Jain, N., Krylov, N.: Large deviations for occupation times of Markov processes with L2 semigroups. Ann. Probab. (36) (5) (2008): 16111641.

[13] Johnson, D.P.: Diffusion approximations for optimal filtering of jump processes and for queueing networks. Thesis (Ph. D.)-University of Wisconsin-Madison, (1983)

[14] Klosek, M.M., Matkowsky, B.J., Schuss, Z.: First-Order Dynamics Driven by Rapid Markovian Jumps. SIAM J. Appl. Math. 49(6) (1989).

[15] Knessl, C., Matkowsky, B.J., Schuss, Z., Tier, C.: An Asymptotic Theory of Large Deviations for Markov Jump Processes. SIAM J. Appl. Math. 46(6) (1985).

[16] Koutsoukos, X.; Riley, D.: Computational Methods for Verification of Stochastic Hybrid Systems. IEEE Trans. on Systems, Man and Cybernetics. Part A. 38(2), 385-396 (2008).

[17] Lygeros, J.: On reachability and minimum cost optimal control. Automatica 40 (6) (2004).

[18] Ma, M., Rockner, M.: "The Theory of (Non-Symmetric) Dirichlet Forms and Markov Processes" Springer Verlag (1990).

[19] Matkowsky, B.J., Schuss, Z.: The exit problem for randomly perturbed dynamical systems. SIAM J. Appl. Math. 33 (1977).

[20] Varadhan, S.R.S.: "Large Deviations and Applications". (Philadelphia: SIAM) (1984).

[21] Yuan-jin Liu, G. Yin: Asymptotic Expansions of Transition Densities for Hybrid Jump-diffusions. Acta Math.Appl. Sinica (20)(1) (2004): 1-18.

[22] Wu, L.: A deviation Inequality for Non-reversible Markov Processes. Annales de l'institut Henri Poincaré (B) Probabilités et Statistiques, (36)(4) (2000): 435-445. 